\title{
LA EDUCACIÓN FÍSICA EN LA EDUCACIÓN INICIAL: EJE FUNDAMENTAL DE CONSTRUCCIÓN DEL SUJETO
}

\author{
Physical Education in the early education: Essential axis of subject building \\ Educação Física na educação infantil: A pedra angular da construção do sujeito \\ Alexander Urrego Gutiérrez \\ Universidad Pedagógica Nacional. Fono: 30452960 70. Correo electrónico: \\ drumsbassdrums@gmail.com
}

\begin{abstract}
Resumen
El presente resumen, da cuenta de un proceso de recolección de datos, consulta bibliográfica y análisis de resultados; en torno a la implementación de una educación inicial, enfatizada en la educación física; entendida esta como aquel acto educativo continuo, que, a partir del cuerpo, construye toda la complejidad del ser humano.

La propuesta nace tras el análisis de las políticas actuales de educación inicial en el país; así como de la observación de algunos puntos relacionados con el mismo tema, propuestos en el borrador del Plan Nacional de Desarrollo 2014-2018.

El problema a tratar está definido por la falta de coherencia entre el discurso y la práctica del gobierno nacional en materia de educación inicial. Prueba de ello, algunos apartes que se desarrollarán en la ponencia; que muestran una aparente preocupación de las autoridades frente a estos temas, plantean algunas opciones para enfrentarlos, proyectan acciones concretas en este sentido; pero al pasar el tiempo, no se reflejan en hechos reales. El objetivo principal del trabajo, es verificar la necesidad de una educación inicial para todos que enfatice en el desarrollo humano, partiendo del desarrollo de la motricidad y pasando por factores, psíquicos, emocionales y sociales que generen en el niño experiencias tendientes a construir un sujeto consciente, crítico y creador. De darse resultados positivos, que verifiquen lo indispensable que puede llegar a ser la educación inicial; se buscará desarrollar una propuesta de mayor alcance, que busque dar solución al problema mencionado en el párrafo anterior.
\end{abstract}

La educación física en la educación inicial: eje fundamental de construcción del sujeto 
Los métodos utilizados para esta investigación son: Hermenéutica o análisis e interpretación de datos. Se trata de una Investigación principalmete cualitativa, sin embargo, también se aportan algunos datos cuantitativos para concretar ciertos puntos. Se proponen y ejecutan algunas sesiones de educación física, con el fin de estimular el desarrollo integral de los niños. También se utiliza Investigación Acción Participante, ya que se elaboran y aplican instrumentos de recolección de información que miden la percepción de los padres y maestros de los niños objeto del estudio, frente al proceso. Se realiza triangulación de datos a partir de los distintos instrumentos aplicados, la observación en diarios de campo y la base teórica consultada. La percepción, tanto de padres, como de maestros frente al proceso de los pequeños; tiende a reivindicar la importancia de la clase de educación física como motor del desarrollo multidimensional del infante. Preliminarmente, se puede concluir que la educación física tiende a propiciar el desarrollo integral del ser humano a partir del cuerpo y el movimiento; que este movimiento debe ser acompañado de un proceso constante de reflexión y representación, es decir, que se debe pasar de lo motor a lo cognitivo-emocional para posteriormente reflejarse en el ámbito social. Fuente de la interacción de los seres humanos, por tanto, motor de su desarrollo.

Palabras clave: educación física; psicomotricidad; desarrollo humano; educación inicial; sujeto.

\begin{abstract}
This abstract, puts forward a data collection process, bibliographic search and results analysis; around an early education implementation process emphasized on Physical education; understood as the chronic educational act, which starting with the human body, it constructs all the human being complexity. The approach was born after the analysis of the current policies for initial education in the country; also through the observation of some issues related to the same topic that was proposed in the National Development Plan's draft. The problem to attend is defined by the inconsistency between the discourse and the praxis from the National Government facing the initial education. As proof of it, there are some stages that will be developed in the paper which show an apparent concern from the La educación física en la educación inicial: eje fundamental de construcción del sujeto
\end{abstract}


authorities regarding these topics. They account for some options to deal with those themes, they project concrete actions on this matter; but by the pass of the time, those actions are not materialized into real facts. The main outcome of this endeavor is to check the necessity of an initial education for everybody that emphasizes on the human development, starting from motor development and going by psychic, emotional and social agents that generate experiences in the child, which tend to build a conscious, critical and creative individual. If it positive results -which verify how the essential initial education can be- come, we will aim to develop a wider proposal that searches to give a solution to the problem mentioned in the last paragraph. The methods used in this research are hermeneutics, or analysis and data interpretation. It is about a qualitative research mainly, however, it brings some quantitative data to specify some specific points. Some physical education sessions are proposed and performed to stimulate the kid's integral development. In the same way it is utilized participatory action research, because data collection instruments that measure the parents' and teachers' Kids' perception in front of the process, are produced and applied. In addition data triangulation is effected from the different instruments implemented: the notes in field diaries and the theoretical base consulted. The parents' and the teachers' perception facing the kid's process; tends to claim the value of the physical education class, as the multidimensional kid development engine. Preliminarily, we can infer that the physical education tends to propitiate the integral development of the human being, coming from the body and the movement and this one should be accompanied of a continuous reflection and representation process, that is to say, we have to pass through the motor skills to the emotional-cognitive, to be later reflected in the social scope which is source of the human being interaction, therefore, engine of their development.

Key Words: physical education; psychomotor; human development; early education; subject.

\section{Resumo}

O presente resumo, leva em conta um processo de recolecção de dados, consulta bibliográfica e analise de resultados, ao redor da implementação de uma educação inicial, enfatizada na educação física, sendo esta entendida como aquele ato educativo continuo, o La educación física en la educación inicial: eje fundamental de construcción del sujeto 
que, a partir do corpo, constrói toda a complexidade do ser humano. O objetivo principal deste trabalho e comprovar a necessidade de uma educação inicial para todos, que tenha ênfase no desenvolvimento humano, partindo do desenvolvimento da motricidade y passando por fatores, psíquicos, emocionais e sociais que gerem na criança experiências tendentes à construção de um sujeito consciente, critico e criador. Si dê-se resultados positivos, que verifiquem o indispensável que pode legar a ser a educação inicial, buscasse desenvolver uma proposta de maior alcance, que busque dar solução ao problema referido no parágrafo anterior.

Os métodos utilizados para esta pesquisa são: hermenêutica o analise e interpretação de dados. Trate-se de uma pesquisa principalmente qualitativa. Aliás, aportam-se alguns dados quantitativos para concretizar certos pontos. Propor-se e executam-se algumas sessões de educação física, para estimular o desenvolvimento integral das crianças. Também, utiliza-se nesta pesquisa Ação Participante, já que se elaboram y aplicam-se instrumentos de recolecção de informação que medem a percepção dos pais e os professores dos meninos objeto de pesquisa, frente ao processo. Realiza-se triangulação de dados desde o distintos instrumentos aplicados, a observação de diários de campo.

Palavras Chaves: educação física; educação inicial; motricidade; sujeito; desenvolvimento humano.

La educación física en la educación inicial: eje fundamental de construcción del sujeto 
“Lo que se les dé a los niños, los niños darán a la sociedad” Karl A. Menninger (1893-

Al observar por distintos medios la evolución que ha tenido el niño en la sociedad, su papel en la familia, en la escuela, incluso en las calles y los parques, se puede visualizar una panorámica de la evolución misma de la sociedad mundial, lo que fácilmente permite analizar la influencia del mundo en los niños y de los niños en el mundo. Como es claro para todos, los niños de hoy, pronto serán adolescentes, saldrán a las calles y elegirán algún camino por el cual transitar su juventud; posteriormente, serán los hombres y mujeres que tendrán las riendas de un país, o serán víctimas de aquellos que tienen el control. Pero, ¿qué tiene que ver la niñez con las condiciones de desigualdad que afectan a una sociedad? Las respuestas para este interrogante son variadas; sin embargo, en esta ocasión se busca ahondar en dos puntos fundamentales del problema. Primero, la niñez es uno de los sectores poblacionales que más sufre violación de derechos, no sólo en materia de salud o educación. Segundo, la descomposición social de países como el nuestro es, probablemente, consecuencia de esta violación.

Pocos dudan que la educación sea una herramienta bastante eficaz, concebida hace siglos por el ser humano para formar al hombre; y a través de ello, dar forma a la sociedad; sin embargo, esta herramienta en manos de intereses oscuros se convierte en un arma de alienación masiva. El gobierno nacional de la República de Colombia, tampoco ignora la importancia de la educación para sus niños y jóvenes; por lo cual ha diseñado, estudiado y aprobado actos legislativos cuyo interés aparente es brindar a todos los niños y jóvenes del país una educación pertinente y de calidad.

Para indagar la legislación colombiana en materia de educación, se tomará como principal referente la Ley 115 de 1994, también llamada Ley General de Educación. En ese documento, el Estado muestra una posición decidida en la búsqueda de una educación accesible para el pueblo colombiano, que beneficie a toda la población en edad escolar y que propenda por el progreso social basado en la inversión y mejoras en materia educativa. En lo sucesivo, se analizarán algunos apartes de la ley en mención, así como de otras 
políticas públicas concernientes al tema, contrastando finalmente sus propuestas con la realidad observada.

El Artículo $5^{\circ}$ de la ley 115 destaca los fines a que atenderá la educación en el país, mencionando en primer lugar el siguiente: "El pleno desarrollo de la personalidad... dentro de un proceso de formación integral, física, psíquica, intelectual, moral, espiritual, social, afectiva, ética, cívica y demás valores humanos". (Congreso de la República de Colombia, 1994). Más adelante, en medio de una concepción de educación física humanizada, se hará un análisis detallado de la relación entre estos aspectos o dimensiones del ser humano y el desarrollo integral.

Los artículos $11^{\circ}$ y $17^{\circ}$, advierten la obligatoriedad de mínimo (también máximo) un grado de educación preescolar, antes de los 6 años de edad. Lo anterior subestima notablemente la importancia de una educación preescolar, asistida por profesionales, constituida mínimo por tres grados, que inicie de manera temprana -lo que no quiere decir prematura-; educación que, a partir de la motricidad, potencialice cada una de esas dimensiones humanas que conforman la integralidad del ser.

El artículo $14^{\circ}$ menciona las áreas del conocimiento obligatorias para las instituciones de educación preescolar, básica y media; se destaca de este artículo el numeral b) que considera áreas obligatorias: “...El aprovechamiento del tiempo libre, el fomento de las diversas culturas, la práctica de la educación física, la recreación y el deporte formativo, para lo cual el Gobierno promoverá y estimulará su difusión y desarrollo”. (Congreso de la República de Colombia, 1994)

Adicionalmente, el párrafo $1^{\circ}$ del mismo artículo menciona: "El estudio de estos temas y la formación en tales valores, salvo los numerales a) $\boldsymbol{y} \boldsymbol{b}$ ), no exige asignatura específica...". (Congreso de la República de Colombia, 1994) De lo anterior, se deduce que todas las instituciones de educación preescolar, además de las de básica (primaria y secundaria) y media; DEBEN ofrecer dentro de sus planes de estudios el área de educación física, orientada por un profesional en dicha materia. Será necesario observar la realidad circundante para comprobar la aplicación de estas normas.

La educación física en la educación inicial: eje fundamental de construcción del sujeto 
Acto seguido, esta benéfica ley define la educación preescolar: “ARTÍCULO 15. Definición de educación preescolar. La educación preescolar corresponde a la ofrecida al niño para su desarrollo integral en los aspectos biológico, cognoscitivo, sicomotriz, socioafectivo y espiritual, a través de experiencias de socialización pedagógicas y recreativas". (Congreso de la República de Colombia, 1994)

Tras leer tal definición, analizar cada uno de los aspectos que ofrece desarrollar e imaginar las innumerables experiencias necesarias para lograr tal fin; no queda más que preguntarse: ¿Es suficiente un año para desarrollar tales aspectos en el niño? ¿No será más benéfico para la sociedad en que vivimos, dedicar un poco más de tiempo para construir seres humanos integrales? Sabiendo que la primera infancia es la edad en la cual se desarrollan aspectos decisivos para el resto de la vida ¿Debería el gobierno nacional institucionalizar la política pública del preescolar de tres grados obligatorio? ¿Qué sería de nuestra sociedad si eso pasara? ¿Es la educación física una herramienta efectiva para lograr estos objetivos?

En el artículo $16^{\circ}$, se describen los objetivos de la educación preescolar; algunos de ellos son: el conocimiento del cuerpo, la lateralidad, la motricidad, desarrollo de la creatividad, habilidades y destrezas, formas de expresión y comunicación, participación en actividades lúdicas, entre muchos otros. Este apartado describe como objetivos de la educación preescolar, asuntos propios del área de la educación física; además, nos puede conducir con toda coherencia a regresarnos a las preguntas del párrafo anterior antes de continuar. No es descabellado pensar en la necesidad de implementar los tres grados de preescolar obligatorio, incluso el gobierno estará de acuerdo.

Su aceptación de esta necesidad está tímidamente expresada en el artículo $18^{\circ}$ con el que se cierra el recorrido por esta ley:

“ARTICULO 18. Ampliación de la atención. El nivel de educación preescolar de tres grados se generalizará en instituciones educativas del Estado o en las instituciones que establezcan programas para la prestación de este servicio. Para tal efecto se tendrá en cuenta que la ampliación de la educación preescolar debe ser gradual a partir del 
cubrimiento del ochenta por ciento $(80 \%)$ del grado obligatorio de preescolar establecido por la Constitución y al menos del ochenta por ciento $(80 \%)$ de la educación básica para la población entre seis (6) y quince (15) años". (Congreso de la República de Colombia, 1994).

Si tenemos en cuenta la condición de cobertura del $80 \%$ en un sólo grado de preescolar, así como en básica y media, y la fecha de entrada en vigencia de esta ley (1994); podemos hacer un diagnóstico, no muy alentador, de la efectividad de las medidas tomadas por el gobierno tras más de 20 años.

La política pública "De cero a siempre" es otra de las medidas adoptadas por el gobierno, esta vez en el Plan Nacional de Desarrollo 2010-2014 Prosperidad para todos. Esta política reconoce la importancia de la primera infancia en el desarrollo del ser humano, así como la necesidad de crear condiciones de equidad para la erradicación de problemas como la pobreza extrema.

En este sentido, el documento oficial de la política pública "De cero a siempre" dice lo siguiente:

"Evidencias científicas demuestran que si bien el desarrollo humano es un proceso continuo que se da a lo largo de la vida, las bases sobre las cuales se irán complejizando las capacidades, habilidades y potencialidades humanas se sientan en los primeros años. El mayor número de conexiones cerebrales, el desarrollo de las habilidades básicas del lenguaje, la motricidad, el pensamiento simbólico, entre otras, así como los cimientos sobre los que se construyen las relaciones consigo mismo, con las demás personas y con el entorno, empiezan a generarse desde el periodo perinatal y tienen su más alto nivel antes de que las niñas y niños ingresen a la educación formal" (Castro \& Vizcaino, 2010)

Teniendo en cuenta que el gobierno nacional es consciente de esta realidad y que además declara en el mismo documento que: “...toda inversión que se concentre en mejorar las condiciones de atención en los primeros años de vida, reduce las necesidades de gasto requeridas para garantizar el cumplimiento de los derechos en etapas posteriores de la vida". (Castro \& Vizcaino, 2010). Es inconcebible que en este documento no exista una 
profundización en el tema educativo y que mucho menos se aborde la educación física como herramienta posibilitadora del desarrollo integral que aparentemente se busca.

Este proyecto, (política pública de cero a siempre), tiene como fundamento la ley 1098 de 2006 o Código de infancia y adolescencia, cuyo artículo $28^{\circ}$ reitera el derecho de los niños a la educación; sin embargo, insiste en la obligatoriedad de sólo un año de preescolar en lugar de los tres considerados desde 1994. Además de reconocer este derecho para todos los niños y jóvenes, en el artículo $29^{\circ}$ se enfatiza en la educación inicial, comprendida entre los cero y los seis años de edad. Finalmente, en el artículo $41^{\circ}$ se enumeran las obligaciones del Estado en materia de infancia y adolescencia; en donde resalta garantizar los derechos de los niños y niñas en materia de formación y desarrollo integral. Habrá que seguir indagando para ver qué tanto ha cumplido el Estado con estas obligaciones.

Para tener una perspectiva del futuro cercano, se dio un vistazo al Plan Nacional de Desarrollo 2014-2018 aún sin sancionar. Allí, se encuentran algunas pistas de las acciones que pretende tomar el gobierno para mejorar la cobertura y la calidad de la educación en el país; sin embargo, aunque queda de manifiesto que no se ignora la importancia de los temas que nos convocan (educación inicial, primera infancia, preescolar de tres grados, educación física) las medidas no son suficientes, adecuadas, ni se cumple con objetivos trazados hace más de 20 años.

En este documento, en el artículo $52^{\circ}$ se define la educación inicial como un derecho impostergable de todos los niños y niñas, previo al ingreso a la educación formal; se anota en el parágrafo $2^{\circ}$ del mismo artículo, un grado de transición obligatorio, muestra del conformismo que existe en el ejecutivo frente al grado único de preescolar con que se cuenta actualmente.

A partir de ahora, la preocupación de esta ponencia se centrará en mostrar algunas de las características más relevantes de la educación física en la educación inicial. Basado en autores de reconocida trayectoria, se verificará la importancia del acompañamiento en el aprendizaje, a través de la experiencia del cuerpo. 
¿Por qué la educación física? Este trabajo busca afirmar la pertinencia de esta disciplina en el proceso de construcción integral del sujeto. Teniendo en cuenta las consideraciones de la legislación en cuanto a la importancia de la primera infancia en el desarrollo integral del ser humano, se puede intuir lo esencial que resulta esta etapa de la vida para la formación de los individuos que integrarán la sociedad del futuro; y no es para menos: en los cinco primeros años de vida se desarrolla casi la totalidad del cerebro, además de la mayor parte de funciones motrices gruesas, así como aspectos de las dimensiones corporal, afectiva, ética, estética, espiritual, comunicativa, psico-social entre otras.

Esta última que se menciona cuenta con dos componentes: la parte psico, que asume la relación del ser humano consigo mismo, y el componente socio, que trata de la relación del hombre con el otro. Estos son pues, los dos tipos de relación que sostiene el ser humano durante su existencia. A través de la mediación del aspecto motriz, que hará tándem con los dos componentes anteriores (psico y socio); se analizarán dos posturas de la educación física como herramienta de desarrollo humano, basada en las relaciones con el propio YO y con los otros YO.

Para G. Lagrange "la Psicomotricidad educa al niño en su globalidad, ya que actúa conjuntamente sobre sus diferentes comportamientos: intelectuales, afectivos, sociales, motores, y psicomotores ayudándole a superar más o menos sus normas, favoreciendo la evolución de su esquema corporal y de su organización perceptiva”. (Escribá, 1999)

Es importante resaltar que según esta teoría, el movimiento es motivado, acompañado y supeditado, generalmente, a un objetivo o meta; es decir, movido por la voluntad, por un deseo de transmisión de ideas, de expresión, de crear; Escribá lo refiere de esta manera: "La función motriz, en definitiva, no es nada sin el aspecto psíquico; por la intervención del psiquismo, el movimiento se convierte en gesto, es decir, en portador de respuesta, de intencionalidad y de significación”. (Escribá, 1999)

Algunas otras características que desarrolla la psicomotricidad son: $L a$ comunicación. Que trata del establecimiento de relaciones significantes entre su cuerpo y el 
mundo exterior, por medio de la emisión y recepción de mensajes, expresiones motrices y verbales que desenvuelven al niño y lo afianzan como ser humano que impacta y es impactado por el mundo.

La creación. De la cual Escribá dice:

“En el juego del niño se pueden distinguir dos aspectos: la imitación de la realidad, que es fruto del aprendizaje; y la contribución personal del niño, en la que pone de manifiesto lo que sabe, cómo lo sabe y de qué forma vive emocionalmente lo que sabe... todos estos hechos se manifiestan por la vía de la expresión motriz lúdica”. (Escribá, 1999)

La descentración. "permite al niño salir de sí mismo y ponerse en el lugar del otro. Para ello es necesario que sea capaz de tomar distancia afectiva de los objetos y de los otros”. (Escribá, 1999). Esta capacidad de ponerse en el lugar de otro, es ampliamente abordada por Daniel Goleman en su obra "La inteligencia emocional" denominándola empatía; término que se verá reflejado en el reporte de experiencias prácticas vividas durante el desarrollo de este trabajo investigativo.

El aspecto socio, se manifiesta en la vivencia y reflexión de situaciones colectivas encontradas en los juegos deportivos, como la cooperación y la oposición; pues esta corriente de la educación física, busca el valor del movimiento en la expresión, interacción, comunicación e intercambio. Encontramos en Parlebas (1984) el término comunicación motriz, que se refiere a la relación dada entre las acciones motrices de distintos sujetos en una situación motriz determinada. (Saraví, 2004)

En la vida adulta, nuestros niños y jóvenes deberán tomar decisiones en aspectos relacionados con cada una de sus dimensiones, esto indica que la dimensión motriz no se encuentra exenta y puede ser determinante en la calidad de vida de una persona; ya que una decisión equivocada en el ámbito motriz puede significar para alguna persona un grave accidente: "Vivenciar distintos tipos de situaciones socio motoras enriquecerá las posibilidades de recepción de información perceptiva y de confrontación con tomas de decisiones motrices múltiples y variadas". (Saraví, 2004) Se entiende además, que en este tipo de relaciones propiciadas en los juegos, cada jugador debe asumir ciertas normas y 
reglas que lo preparan para adaptarse a los lineamientos de la vida en sociedad. Sin embargo, mencionan Caruso y Dussel que "estas reglas pueden ser modificadas o replanteadas por los sujetos y de esa manera pueden otorgar nuevos significados a su mundo" (Caruso \& Dusel, 1996). En esta teoría socio motricista, también conocida como praxiología motriz, Pierre Parlebas busca mostrar que existen formas de interacción humana diferentes y más provechosas que la competencia.

Para cerrar el capítulo que da cuenta de la labor que cumple la educación física en la formación de un sujeto desarrollado de manera integral, es necesario re-unir los conceptos divididos anteriormente: Psico y socio. Al recordar las características de cada componente y reunirlas, nos encontramos con que efectivamente se involucra la relación del ser consigo mismo y con los otros seres humanos que le rodean; estos dos tipos de relación, como ya se mencionó son los que nutren las distintas dimensiones del ser humano. Dimensiones que, al satisfacer sus necesidades; acercarán al hombre un poco más al completo desarrollo de su potencial, al desarrollo humano.

Para concluir con este punto, baste hacer la aclaración definitiva desde las palabras del profesor Jorge Saraví: "Sociomotriz es toda aquella situación en la que interactuamos motrizmente con otros — sean ellos adversarios o compañeros, o ambos a la vez- y, como contrapartida, psicomotor es lo referido a aquel sujeto que se mueve, que se desenvuelve en solitario". (Saraví, 2004)

A continuación, trasladamos el eje de análisis a una experiencia concreta, vivida por el ponente; que guarda total relación con el tema que se desarrolla. A partir de la teoría mencionada, las observaciones de campo realizadas por el profesor y los resultados arrojados por una encuesta aplicada a padres y docentes del jardín infantil denominado The Kids House Preschool; -institución en donde se desarrolla la mencionada experiencia- se intentará demostrar el nivel de importancia que realmente demanda la educación inicial o de la primera infancia.

The Kids House Preschool, es un jardín infantil de carácter privado, ubicado en el barrio Modelo Norte de la localidad de Barrios Unidos en la ciudad de Bogotá. Allí, asisten 
niños entre los 3 y los 6 años de edad, que cursan alguno de los tres grados sugeridos para una adecuada educación inicial; denominados en este caso: Párvulos, pre jardín y Jardín.

Estos niños, en su mayoría forman parte de familias de estrato 3, 4 o mayores; hecho que les permite a sus padres pagar considerables sumas mensuales a cambio de una formación inicial de calidad, que propenda por el desarrollo integral de sus hijos en busca de un mejor mañana. Cabe preguntarnos aquí, ¿Y los niños de estrato 1 y 2 , cuyos padres no pueden pagar? Teniendo en cuenta la gran cantidad de niños que viven en estos estratos, parece preocupante imaginar el futuro de nuestro país.

Además de la importancia del desarrollo motriz, determinante en la construcción de otras dimensiones del ser humano; la experiencia en esta institución educativa, ha aportado importantes hallazgos en cuanto a la construcción de las dimensiones social, emocional y cognitiva de los pequeños. La experiencia que se menciona consta de la implementación de una serie de sesiones, basadas, entre otras cosas, en las teorías psicomotricista y sociomotricista. Estas sesiones han sido planeadas y ejecutadas con la intención de potencializar las capacidades, habilidades, destrezas; y formas de comunicar, interpretar y sentir de los pequeños. Además, cada sesión ha sido retroalimentada a partir de apuntes tomados durante su transcurso y tras su finalización. A continuación, se mencionan algunos ejemplos concretos observados y sistematizados durante el proceso.

Frente a la dimensión social, en primer lugar se puede mencionar la actividad de cierre de todas las sesiones. En cada clase, al finalizar, se hace una pequeña reunión en mesa redonda en la cual se habla acerca de las experiencias vividas por los niños. Es ahí en donde ellos tienen sus primeros acercamientos al uso de la palabra dentro de un grupo de pares, aprenden entre otras cosas a escuchar y respetar la palabra de los otros y a comunicar sus ideas de forma verbal. En este espacio, además, es posible ejercitar la memoria por medio de la remembranza de los sucesos vivenciados y su relación con la vida cotidiana (capacidad asociativa).

Durante la sesión número cuatro, llevada a cabo en marzo de 2015, se realizan ejercicios que movilizan la expresión corporal y el lenguaje no verbal. En estas actividades, 
se provee a los niños de experiencias que les permitan transmitir e interpretar mensajes, significados y significantes con su cuerpo hablante y su boca silente. Esta capacidad del ser humano de comunicar con su lenguaje corporal, cobra importancia a través de los años, en el establecimiento de las distintas relaciones sociales.

Aunque suene extraño o pretencioso, en una de las sesiones desarrolladas en el jardín tuvo lugar una pequeña lección de democracia:

Tras el reiterativo mal comportamiento de uno de los chicos, el profesor optó por retirarlo del gimnasio momentáneamente. Cuando se disponía a conducirlo a su aula habitual, uno de los niños que aguardaba el desenlace de la historia, se puso de pie y gritó: “¡No es justo!”... tras escuchar esta exclamación, el profesor se volvió hacia él y le preguntó a qué se refería. El estudiante explicó que no le parecía justo que su compañero fuera retirado de la clase. Esta actitud, motivó al profesor a reconsiderar su decisión y plantear una alternativa. Propuso entonces a los niños realizar una votación para decidir si el chico debía o no ser retirado de la clase. Finalmente, por mayoría de votos se decidió que el niño podría continuar en la clase, pero con el compromiso de no interrumpir más su desarrollo.

De esta manera, los chicos ejercieron el voto, derecho constitucional que les permitirá en su vida adulta decidir sobre el futuro de su sociedad. En este caso decidieron sobre su pequeño grupo social y vigilaron además, el cumplimiento del compromiso adquirido por parte del infractor.

En este mismo caso, vemos también una clara señal de empatía, una de las características fundamentales de la inteligencia emocional; postulado de Daniel Goleman. Esta cualidad se refleja claramente en el niño que, poniéndose en el lugar de su compañero y entendiendo lo triste que lo hacía sentir el ser retirado de la clase; intercedió ante el profesor por él.

En las actividades de expresión corporal de la mencionada clase número cuatro, se evidenciaron dos importantes aspectos del área cognitiva del ser humano: la creatividad y la imaginación. Estos son susceptibles de potencialización principalmente en la primera 
infancia, pues por medio de sus movimientos, los niños crean diferentes formas de vivir una situación imaginaria, concebida por algunos instantes como real.

Estos son sólo algunos ejemplos de la gran variedad de situaciones: propiamente motrices, causa, o efecto de las mismas; que se han dado en medio de las sesiones realizadas en el jardín a lo largo de estos seis meses. Las observaciones que se han compartido en este documento, constituyen el análisis cualitativo de los resultados ofrecidos por la experiencia; adicionalmente, se ofrecen a continuación algunos datos cuantitativos que darán cuenta de la percepción de padres y maestros sobre el proceso de desarrollo de los niños.

La primera encuesta, realizada a dos de las profesoras del jardín; refleja su percepción del proceso de los niños dentro del contexto escolar (o preescolar), es allí donde el juego con sus pares estimula toda la capacidad de movimiento que tiene el infante en su interior. Además, las maestras tienen constante acceso y comunicación con los aspectos cognitivos y emocionales de los estudiantes; lo que hace de su percepción, una sugerente fuente de datos valiosos para nuestro propósito.

Las maestras encuentran un aumento en la frecuencia con la que los niños implementan en su cotidianidad algunos patrones básicos o habilidades motrices, entre ellas: correr, saltar y realizar equilibrio. También se muestran de acuerdo en que los niños han desarrollado mejores hábitos de conducta, expresados en la capacidad de escucha atenta por un tiempo más prolongado. Coinciden también en la observación de un avance significativo durante el tiempo de este estudio, de dos procesos cognitivos importantes para el desarrollo integral de los chicos: la creatividad y la curiosidad. Además mencionan individualmente otros aspectos con alta incidencia como la imaginación y la comprensión.

De forma unánime encuentran también que los niños presentan una mayor fluidez verbal al momento de expresar sus ideas; una de ellas acusa además, mayor fluidez corporal y adquisición de repertorio lingüístico, mientras la otra evidencia en sus chicos mayor coherencia en la expresión de sus ideas. También se manifiestan de acuerdo en que ha mejorado la capacidad de recordar hechos, situaciones o experiencias de aprendizaje. 
Muestran también los resultados de la encuesta una altísima frecuencia de actitudes que reflejan sentimientos de alegría en los pequeños.

Se refleja también en las encuestas alta incidencia de actitudes amistosas, amables y respetuosas en el trato cotidiano de los niños, entre ellos y con las docentes. Finalmente, en la forma 002 -encuesta para profesoras- queda en evidencia la unanimidad de las consultadas en cuanto a tres puntos importantes. Primero, reconocen la participación activa de los niños en la toma de pequeñas decisiones que los afectan directamente; segundo, observan actitudes de empatía en las relaciones entre los estudiantes; y por último, aceptan la necesidad imperiosa del establecimiento del preescolar de tres grados como política pública educativa en todo el país.

La encuesta aplicada a los padres: forma 001, comienza indagando acerca del desarrollo biológico de los niños. Con una muestra de 11 padres de familia consultados, el $63.63 \%$ ( 7 padres) encuentra cambios en la contextura física de sus hijos; principalmente reflejados en la estatura o el peso. Una cifra superior al 50\% de los padres consultados nota desarrollos importantes en la calidad o frecuencia de la utilización de estos patrones básicos o habilidades motrices básicas en los niños: caminar, correr, saltar, coordinar, equilibrarse y patear. De las opciones propuestas, sólo una obtuvo menos del $50 \%$ de incidencia y fue el lanzamiento de objetos. (Por ejemplo pelotas).

El $90.9 \%$ de los padres consultados expresa mejoras en la capacidad de prestar atención y de los hábitos de conducta de sus hijos; el $72.72 \%$ refieren mejor capacidad de escucha. Entre el $72 \%$ y el $91 \%$ de los padres reconocen desarrollos obtenidos durante el tiempo de intervención en cuatro aspectos fundamentales de la dimensión cognitiva del niño, estos son: Imaginación, creatividad, comprensión y curiosidad.

También el 90.9\% expresa la mejora de la fluidez verbal percibida por los padres, en las relaciones comunicativas de sus hijos; además el $63.63 \%$ advierte mayor fluidez corporal y un repertorio lingüístico cada vez más amplio. Más del $80 \%$ de los padres reconoce mayor coherencia en la expresión de ideas. Los once padres encuestados aseguran mejoras en la capacidad de memoria de los niños.

La educación física en la educación inicial: eje fundamental de construcción del sujeto 
Existe una relación interesante en la percepción del área emocional de los estudiantes, por parte de sus padres; mientras el 100\% reconoce aumento de la frecuencia de expresiones de alegría, un contundente $0 \%$ acusa manifestaciones de tristeza reiterativas. Otros bajos porcentajes se presentan frente a actitudes negativas: tan sólo el 18.18\% encuentra expresiones de enojo, y sólo un 9.09\% identifica expresiones de timidez. El $36.36 \%$ nota con frecuencia tranquilidad en sus hijos, mientras el $63.63 \%$ los encuentra inquietos frecuentemente.

Al indagar por actitudes propias de las relaciones familiares de los pequeños, se encuentran los siguientes resultados: el total de los padres reconoce expresiones cariñosas más frecuentes de parte de sus hijos, 90.9\% los encuentra más comunicativos, el 63.63\% los nota confiados en su núcleo familiar, 54.54\% reconoce más actitudes de solidaridad frente a las tareas y situaciones propias del hogar; el $36.36 \%$ reconoce expresiones de comodidad de los niños en el medio familiar y el $27.27 \%$ evidencia apropiación del valor del respeto.

Frente a las relaciones sociales externas al hogar, las encuestas arrojaron estas cifras: $18.18 \%$ advierte actitudes de desconfianza e indiferencia frente a las personas ajenas al núcleo familiar. El $63.63 \%$ observa respeto y cortesía en las relaciones sociales de los pequeños, mientras que el $81.81 \%$ los percibe amables y amistosos.

También el $81.81 \%$ reconoce actitudes de participación de los niños en las decisiones que los afectan directamente, así como actitudes de empatía frente a los sentimientos ajenos. Por último, el $90.9 \%$ de los padres apoyan la idea de la obligatoriedad del preescolar de tres grados para los niños en su primera infancia.

Encontramos todos estos resultados y hallazgos aptos para defender la educación física, como herramienta indispensable en la formación integral de nuestros niños y niñas; así como para argumentar la importancia de una educación ofrecida desde los albores de la primera infancia, enfatizada en los diferentes desarrollos que propicia la educación física. Tras la implementación de cada una de las sesiones, se percibe en el comportamiento de los niños una mayor disposición a cumplir las reglas de juego, las normas establecidas, códigos 
éticos y de conducta; además de muchas expresiones de emoción y alegría frente a la posibilidad de aprender jugando.

Para argumentar esta proposición, en la que los comportamientos, actitudes y formación de valores en los niños, encuentra sus bases; es preciso citar a un importante referente en materia de relaciones sociales:

"La actividad que asegura el aprendizaje y la adquisición de aptitudes o tipos de comportamiento se desarrolla allí por medio de un conjunto completo de comunicaciones reguladas (lecciones, preguntas y respuestas, órdenes, exhortaciones, signos codificados de obediencia...) y por medio de una serie completa de procesos de poder”. (Foucault, 1998)

Encontramos entonces, que los procesos de formación del ser humano están atravesados por las acciones que tienen incidencia sobre su vida; ya sea de manera intencional o no. En este punto, es importante resaltar que los mencionados procesos de poder no se refieren a estrategias represivas o de coacción; sino más bien se trata de acciones ejercidas sobre las acciones conductuales de otros, en este caso de los niños. Es allí donde el papel del maestro tiene vital importancia, pues es desde esta posición que se pueden planear y ejecutar acciones intencionadas, aplicables sobre las acciones de los pequeños, en busca de una repercusión significativa en sus procesos educativos y de aprendizaje.

Esta intervención sobre la construcción de un ser humano, implica una responsabilidad inconmensurable para el docente; pues de la calidad de la formación impartida puede depender en gran medida el desarrollo integral de la sociedad del futuro. En este mismo sentido:

“...Foucault considera que cada época cultural posee un código fundamental... -que llama episteme- ... y sobre cuyo fondo se elaboran, piensan e interpretan los objetos... explicita que los códigos fundamentales de una cultura, los que rigen su lenguaje, sus percepciones, sus cambios, sus valores, sus técnicas, la jerarquización de sus prácticas, fijan previamente para cada hombre los órdenes empíricos con los que tendrá algo que ver y dentro de los que se reconocerá" (Ávila, 2007)

Sin embargo, es importante mencionar que la formación que se pretende defender en este trabajo no es de ninguna manera totalitaria, represiva o dominante; por el contrario, se La educación física en la educación inicial: eje fundamental de construcción del sujeto 
busca a través de las experiencias proporcionadas a los niños; motivar una actitud crítica, reflexiva y consciente; que propenda por la libertad y la autonomía del individuo en medio de su grupo social.

"Cuando se define el ejercicio del poder como un modo de acción sobre las acciones de otros... se incluye un elemento importante: La libertad. El poder se ejerce solo sobre sujetos libres y solamente en la medida en que ellos son libres... sujetos individuales o colectivos que están enfrentados con un campo de posibilidades en el que se puedan realizar diversas formas de conducirse, diversas reacciones y diversos comportamientos". (Foucault, 1998)

Ahora bien, es importante aclarar el uso que se le está dando al término sujeto en este texto; ya que es aquél en quien recae la acción formativa, -La educación física en la educación inicial- y quien dará cuenta de los resultados de la propuesta que se presenta a lo largo del presente documento. Al respecto, el pensador francés advierte: "Existen dos significados de la palabra sujeto: sujeto a alguien por el control y la dependencia, y el de ligado a su propia identidad por una consciencia o autoconocimiento" (Foucault, 1998)

Parece claro entonces que el significado del término -sujeto- dentro de este texto es el segundo. Desde esta propuesta se busca formar sujetos dotados de una identidad propia: colectiva, gracias a una consciencia de las dinámicas sociales, políticas, económicas y culturales que los rodean; pero además, individual, tras un conocimiento pleno de sí mismo, de sus emociones, sensaciones, percepciones, hábitos y posibilidades de acción dentro del mundo en el que se encuentran.

Adicionalmente, se considera decisivo que el proceso que inicia en la edad preescolar sea continuado y fortalecido a lo largo de todas las etapas de la vida del sujeto; teniendo en cuenta que la educación es un proceso permanente e inacabado.

“...Es importante recordar que las prácticas lúdicas... son acciones desarrolladas por los grupos humanos... que satisfacen necesidades emocionales y socio-afectivas de los sujetos. Desde una perspectiva sociológica y antropológica, las prácticas lúdicas constituyen reproducciones simbólicas de la sociedad en contextos culturales específicos, en las cuales se expresan, o se contienen, imaginarios culturales y diferentes formas de recreación fantástica de la conciencia”. (Díaz, 2006)

La educación física en la educación inicial: eje fundamental de construcción del sujeto 
De esta manera se verifica, al menos en el caso particular que se ha estudiado; la trascendencia de brindar a los niños y niñas del país, la oportunidad de recibir una formación inicial un poco más extensa. Esta necesidad es reconocida por el gobierno nacional, en la forma de preescolar de tres (3) grados; pero no se observan medidas concretas que propendan por hacer de esta necesidad un caso resuelto.

En el mismo sentido, además de brindar de manera pública, gratuita y de calidad estos tres grados; se considera importante desde esta postura, que el énfasis de dichos ciclos educativos sea la educación física; que desde las diferentes tendencias relacionadas con la educación infantil, muestra su aptitud para la formación de otras dimensiones del ser, como la emocional o la cognitiva; logrando de esta manera una verdadera educación integral.

El objetivo de este trabajo será logrado, tal vez, dentro de muchos años. Es probable incluso, que quien lo realiza no logre ver esa utópica sociedad que se podría construir de tomarse en cuenta la propuesta. Es claro que en el recorrido serán necesarios algunos ajustes, pertinentes en pro de la optimización de los procesos y la consecución de los resultados. La sensación será sin duda gratificante para quien escribe, si logra ver en vida, al menos un inicio expresado en acciones tangibles, de la implementación de este proyecto a nivel estatal.

\section{Referencias bibliográficas}

Ávila, F. (2007). El concepto de poder en Michel Foucault. A Parte Rei, 53, 1-16.

Caruso, M., \& Dusel, I. (1996). “Yo, tú, él: ¿quién es el sujeto?’. En De Sarmiento a Los Simpsons, Cinco conceptos para pensar la educación contemporánea. Buenos Aires: kapelusz.

Castro, A. 1., \& Vizcaino, J. R. (2010). De cero a Siempre. Política pública De Cero a Siempre. Bogotá, Colombia.

Congreso de la República de Colombia. (8 de febrero de 1994). Ley General de Educación, Ley 115 de 1994. Bogotá, Colombia. 
Díaz, H. Á. (2006). La función lúdica del sujeto. UNa interpretación teórica para transformar las prácticas pedagógicas. Bogotá: Cooperativa editorial Magisterio.

Escribá, A. (1999). Psicomotricidad. Fundamentos aplicables a la práctica. Madrid, España: Gymnos.

Foucault, M. (1998). El Sujeto y el Poder. Obtenido de www,philosophia.cl: www,philosophia.cl/escueladefilosofiauniversidadarcis

Saraví, J. (2004). Praxiología motriz y Educación Física, una mirada crítica. Obtenido de http://www.academia.edu/: http://www.academia.edu/1175646/PRAXIOLOG\%C3\%8DA_MOTRIZ_Y_EDU CACI\%C3\%93N_F\%C3\%8DSICA_UNA_MIRADA_CR\%C3\%8DTICA 